\title{
Stiffness of Human Hair Correlates with the Fractions of Cortical Cell Types
}

\author{
Yusuke Ezawa $^{1}$, Shinobu Nagase ${ }^{1}$, Akira Mamada ${ }^{2}$ (), Shigeto Inoue ${ }^{3}$, Kenzo Koike ${ }^{1}(\mathbb{D}$ and \\ Takashi Itou $1, *$ (D) \\ 1 R\&D—Hair Care Products Research, Kao Corporation, 2-1-3, Bunka, Sumida-ku, Tokyo 131-8501, Japan; \\ ezawa.yuusuke@kao.com (Y.E.); nagase.shinobu@kao.com (S.N.); koike.kenzo@kao.com (K.K.) \\ 2 Product Quality Management, Kao Corporation, 2-1-3, Bunka, Sumida-ku, Tokyo 131-8501, Japan; \\ mamada.akira@kao.com \\ 3 R\&D-Analytical Science Research, Kao Corporation, 1334, Minato, Wakayama-shi, \\ Wakayama 640-0112, Japan; inoue.shigeto@kao.com \\ * Correspondence: itou.takashi1@kao.com; Tel.: +81-3-5630-9972
}

Received: 30 January 2019; Accepted: 21 March 2019; Published: 1 April 2019

\begin{abstract}
Background: The objective of this work was to elucidate the hair microstructure which correlates with the stiffness of human hair fibers. (2) Methods: Bending moduli of hair fibers were evaluated for the hair samples from 156 Japanese female subjects. Hair transverse sections were dual-stained with fluorescent dyes which can stain para- and ortho-like cortical cells separately, and observed under a fluorescence light microscope. Atomic force microscopy nanoindentation measurements were performed to examine the modulus inside macrofibrils. (3) Results: The difference in bending moduli between the maximum and the minimum values was more than double. The hair of high bending modulus was rich in para-like cortical cells and the bending modulus significantly correlated with the fraction of para-like cortical cells to the whole cortex. On the other hand, the elastic moduli inside macrofibrils were almost same for the para- and ortho-like cortical cells. (4) Conclusions: Hair bending modulus depends on the fractions of the constitutional cortical cell types. The contribution of the intermacrofibrillar materials, which differed in their morphologies and amounts of para- and ortho-like cortical cells, is plausible as a cause of the difference in the modulus of the cortical cell types.
\end{abstract}

Keywords: AFM nanoindentation; bending modulus; cortical cell; human hair; fluorescence light microscopy

\section{Introduction}

Human hair and wool, two kinds of keratin fibers, are covered with cuticle scales, and the cores mostly consist of cortices. Cortical cells constituting the cortex are composed of rod-like intermediate filaments (IFs) and keratin associated proteins (KAPs) existing around and supporting the IFs. IFs are arranged in a cross-section of assemblies of likely 32 keratin molecules aligned longitudinally [1,2]. In contrast, KAPs are amorphous cysteine-rich proteins and are known to have many variations [3,4] Hair is a composite structure and its physicochemical properties are determined by its main morphological components, the cuticle and the cortex, and, in turn, by the micro-structures and the intra- and inter-molecular interactions of the constituent elements of them, such as IFs and KAPs.

It is known that different types of cortices exist in wool [5] and that wool fibers having a strong curl shape such as Merino wool have a bilateral structure of paracortex and orthocortex existing in the concave and the convex side of the curl, respectively [6]. The IF arrangements inside them are greatly 
different [7]. IFs align almost parallel with one another to the fiber axis in para- and meso-cortical cells and, as a result, a dot patterns are observed in cross section by transmission electron microscope (TEM). Meso-cortical cells further show a pattern of regular hexagonal close packing alignment. On the contrary, whorl patterns are observed by TEM in ortho-cortical cells, and through three-dimensional TEM measurements, it has been confirmed that IFs align helically in ortho-cortical cells of wool [8].

It has been shown for human hairs that para-like, meso-like, and ortho-like cortical cells also exist $[9,10]$ and the IFs helically arrange in the ortho-like cortical cells as well as the orthocortex of wool [9]. Para-like cortical cells locate in the concave side and ortho-like cortical cells in the convex side of the curl for human curly hairs [11]. Bryson et al. [12] have shown in detail the difference in cell distribution between curly hairs and straight hairs by fluorescence light microscopy (FLM) and two- and three-dimensional TEM, and proposed a mechanism for curliness based on the cell type distribution. Through small angle X-ray scattering measurement on Merino wool and human curly hairs using a synchrotron radiation micro beam X-ray of about $5 \mu \mathrm{m}$ diameter, Kajiura et al. [13,14] have shown that the tilt angle of IFs against the fiber axis is small in the cortex at the concave side of the curl and, conversely, rather large at the convex side of the curl. According to their findings, the curl strength of human hair and wool depends on the degree of the inhomogeneity of the internal nanostructure, regardless of ethnic origins [14]. These facts indicate that the macroscopic hair shape relates to the microscopic distribution of the different cortical cell types.

There have, however, been no reports about the relation between microscopic properties and hair stiffness, one of the recognizable physical properties. The hair stiffness depends simply on its diameter. It also relates to the elasticity of the cuticle and cortex and, to be more specific, it should depend on the structure and fraction of their constituents. It has been shown that the bending elastic moduli of hairs from Japanese and Caucasian females are almost constant but, for senior donors who were conscious of the decrease in hair volume or bounce with aging, the moduli of their hairs are relatively lower [15]. It is unclear to which structure the difference in the elastic modulus is attributed, and knowing this would be important to understand practical characters that concern aged persons.

This study focuses on the hair stiffness to understand the structure relating to the bending modulus from a microscopic point of view. Through evaluations of bending elasticity and FLM, it has been found that the bending modulus depends on the fraction of the para-like cortex to the whole cortex. The possible reason for the modulus difference between the para- and ortho-like cortices will be discussed based on the difference in their subcomponents and constitutional molecules.

\section{Materials and Methods}

\subsection{Hair Samples}

About 80 hair fibers from each of 156 Japanese female subjects between 10-70 years old were cut very close to the scalp by a trained beautician. All the subjects had not had a perm within the last six months. The hairs were cut randomly from the top part of the head. Segments of the hairs $3 \mathrm{~cm}$ in length from the root end were used for the experiments in order to avoid property changes due to daily hair damage. African-American hair with a very strong curl shape was used since it was expected to show a clear separation of ortho-like and para-like cortical cells. Hair fibers donated by an African-American male subject were used. The hairs were again cut randomly very close to the scalp and had no history of perm, relaxer, or hair dye treatments.

\subsection{Hair Diameter}

Hair samples were left in a room controlled at $20{ }^{\circ} \mathrm{C}$ and $65 \%$ relative humidity (RH) overnight, and then used for the diameter and bending stress measurements in the same room.

Hair diameters were measured for 30 hair fibers selected randomly from each subject with a rotating fiber diameter system equipped with a laser (Kato Tech Co. Ltd., Kyoto, Japan) on segments 1.0 to $1.4 \mathrm{~cm}$ from the root end for each sample. The orthogonal projection of the hair was measured 
while it rotated at intervals of 30 degrees. The minimum value was taken as the minor axis and the maximum as the major axis at the measured position. Each fiber was measured at five positions at intervals of $1 \mathrm{~mm}$ along the fiber, and the mean values of the minor and major axes were taken as the diameter of the minor axis $(a)$ and that of the major axis $(b)$ of the sample, respectively. The ellipticity calculated from the mean minor and major axes for each subject was $1.23 \pm 0.08$ on average and had no age dependence, similar to the previous results [16].

\subsection{Bending Elasticity}

Hair bending stress was measured with a bending testing machine (KES-FB-2, Kato Tech Ltd., Kyoto, Japan) at $20^{\circ} \mathrm{C}$ and under $65 \% \mathrm{RH}$, according to the method by Mamada and Nakamura [15] except that 30 hair samples were used for each measurement in this study. In brief, thirty hair fibers were perpendicularly affixed between the two pieces of cross-section papers (length: $32 \mathrm{~mm}$, width: $15 \mathrm{~mm}$ ), at $1 \mathrm{~mm}$ interval, with double-sided adhesive tape. The measured part of the samples was in the position range of 1 to $2 \mathrm{~cm}$ from the root ends. A bending angle of 2.5 radians was chosen. Bending stress was measured as a function of curvature and, the bending elasticity per fiber, $B$, was determined from the slope of a stress-strain curve in the curvature range of 1 to $2 \mathrm{~cm}^{-1}$.

From B and the moment of inertia $(I)$ calculated from the averaged radii of the minor axis $(<a>)$ and major axis $(<b>)$ for the used 30 hair samples, bending modulus $(E)$ was determined according to Equations (1) and (2).

$$
\begin{gathered}
B=E \times I \\
I=\pi<a>^{3}<b>/ 4
\end{gathered}
$$

\subsection{Fluorescence Light Microscopy (FLM) of Cortical Cell Types}

Hair samples were immersed in ethanol for one minute to clean their surface and dry, then put in embedding molds. Hair samples embedded in Spurr's resin (Polysciences, Inc., Warrington, PA, USA) were cut into round slices of $5 \mu \mathrm{m}$ in thickness with a microtome (Leichert Ultracut N; Leica Microsystems, Wetzlar, Germany) and then the slices were put on a slide glass. Double staining with fluorescein sodium (FS) and sulforhodamine 101 (SR) were performed [12]. The colors of FS and SR are green and red, respectively. The slices were immersed in a few drops of phosphate-buffered $0.002 \%$ FS solution, left for $18 \mathrm{~h}$ under conditions of dark and high humidity so as not to dry, rinsed by deionized water 6 times, and dried naturally for $20 \mathrm{~min}$. Then, the slices were immersed in a few drops of phosphate-buffered $0.005 \%$ SR solution, left for $1.5 \mathrm{~h}$ under the same condition above, rinsed by deionized water 6 times, and naturally dried. A fluorescence stereoscopic microscope (AxioVision 4.3; Zeiss, Oberkochen, Germany) with a fluorescein filter set (BP450-490, FT510, LP520) was used. The images were digitally recorded and analyzed with analytical software ImageJ [17].

\subsection{Atomic Force Microscopy (AFM)}

Hair samples and a standard fiber were embedded between two pieces of celluloid plate (Sump plate; SUMP Laboratory, Tokyo, Japan) with ethyl acetate. The embedded hair samples and standard fiber were cut by a microtome (EM UC7i, Leica Microsystems, Wetzlar, Germany) with a SYM knife (SYNTEC Co., Ltd., Yokohama, Japan) to obtain smooth fiber cross-sections for AFM. For a standard fiber, we used a fishing line made by fluorocarbon (Seaguar Keigoku No. 2, Kureha Gohsen Co., Ltd., Mibu, Japan) of which Young's modulus had been examined to be $2.9 \pm 0.2$ GPa by a tensile tester (NMB TG-500N; MinebeaMitsumi Inc., Tokyo, Japan) with a speed of $20 \mathrm{~mm} / \mathrm{min}$ in the condition of $20{ }^{\circ} \mathrm{C}$ and $65 \%$ RH.

MFP-3D (Asylum Research, Santa Barbara, CA, USA) equipped with a closed cell to control humidity condition and cantilevers of HQ300-Au (Oxford Instruments plc, Abingdon, UK) were used. The spring constant used in this study was determined to be $25.2 \mathrm{~N} / \mathrm{m}$ by the thermal noise method. Humidity was controlled to be $65.5 \pm 0.5 \%$ RH through use of a saturated solution of sodium 
hydrogen sulphite monohydrate. Samples were left for at least one hour to equilibrate prior to starting measurements. AFM experiments were carried out at $27.1 \pm 0.2{ }^{\circ} \mathrm{C}$.

Firstly, an image of a cross section was obtained in AC mode on a $5 \times 5 \mu \mathrm{m}$ area to determine the positions where the following nanoindentation measurements were performed. Secondly, the AFM was operated in the contact mode and force curves at about the center of macrofibrils were acquired. The loading rate was $1 \mathrm{~nm} / \mathrm{s}$, and the maximum force and the maximum indent depth were less than $300 \mathrm{nN}$ and $8 \mathrm{~nm}$, respectively. More than 100 force curves were obtained for each cortical cell type in the same condition. For the standard fiber, the center part of the fiber was examined for the force curve measurements.

Force curves were analyzed using the Hertz model for a punch indenter by fitting the straight-line portion of Force-Indentation curve in the range of $20 \%-80 \%$ of indentation (ca. $2.5-6 \mathrm{~nm}$ in depth). Firstly, the force curves of the embedded standard fiber were analyzed. The cantilever radius was determined so that the modulus might correspond to the value of Young's modulus ( $2.9 \mathrm{GPa})$. In the calculation, a value of 0.5 for the Poisson's ratio was assumed. The radius of the cantilever was determined to be ca. $6 \mathrm{~nm}$, which is reasonable from the geometry of the cantilever. The cantilever radius values thus determined were used to calculate the elastic moduli of the hair fibers. Again, a value of 0.5 for the Poisson's ratio was assumed for hair [18].

\section{Results}

\subsection{Bending Elasticity}

Figure 1 illustrates the bending modulus $E$ plotted against the age of the subjects. There is no significant age dependence and $E$ values have some variation as previously reported [15]. The difference between the maximum and the minimum values is more than double. Since $E$ is a mean value of randomly selected 30 hair fibers, the large difference in $E$ is meaningful even if Figure 1 shows some scatter. This suggests a non-uniformity within the constituents of hair and that bending stress is, therefore, not determined solely by the hair diameter.

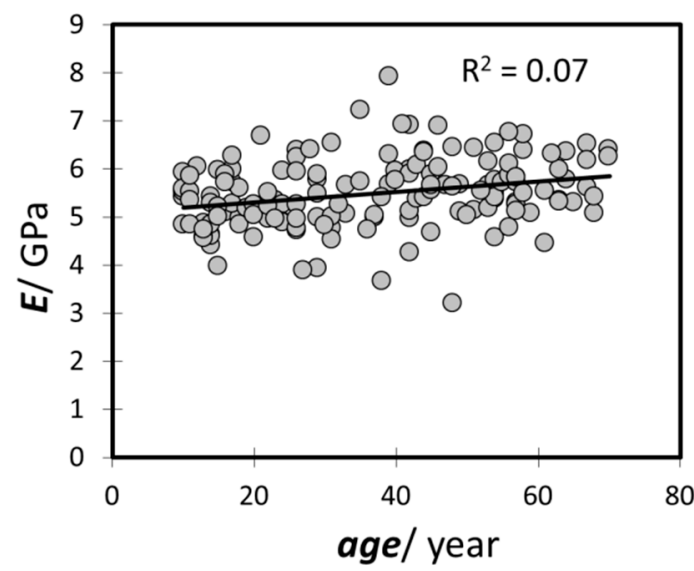

Figure 1. Hair bending modulus vs. age of subjects. Each data point is a mean value of randomly selected 30 hair fibers from Japanese females.

\subsection{Observation of Cortical Cell Types in Hairs of Various Elastic Moduli}

The cross sections of the hair samples having different bending moduli were observed by FLM following a dual fluorescence staining procedure. Figure 2a shows a sample of high bending modulus ( $E=6.5 \mathrm{GPa}$ ) with most of the cortical parts in the hair cross section are stained by FS (green). On the other hand, Figure $2 \mathrm{~b}$ shows a sample of low bending modulus ( $E=4.7 \mathrm{GPa})$, which was mostly stained by SR (red). In addition, Figure 2c shows a sample with bending modulus close to the average 
( $E=5.7 \mathrm{GPa}$ ) with sections stained by both FS and SR, which was the most frequent case for the hair samples observed by FLM.

From FLM on Japanese hairs and by the comparison with TEM observation using the adjacent fiber section, Bryson et al. [12] determined that the parts stained by FS are where para-like cortical cells are located and those stained by SR are where ortho-like cortical cells are located. Applying these results to the present study, it suggests that para-like cortical cells are rich in the hair of high bending modulus and, conversely, ortho-like cortical cells are rich in the hair of low bending modulus.

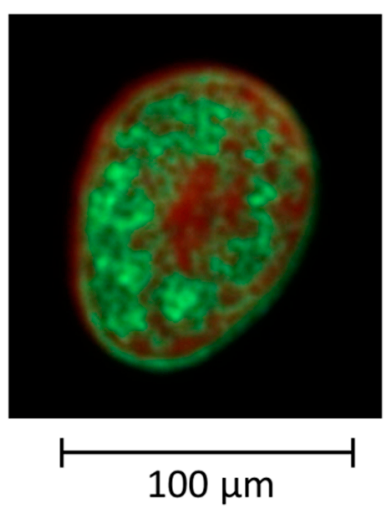

(a)

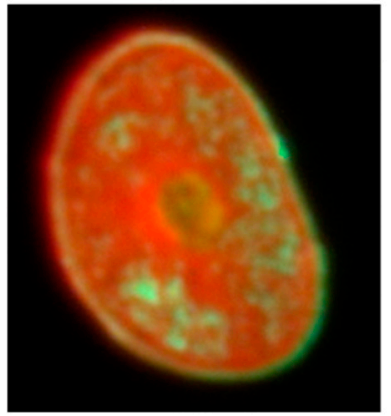

(b)

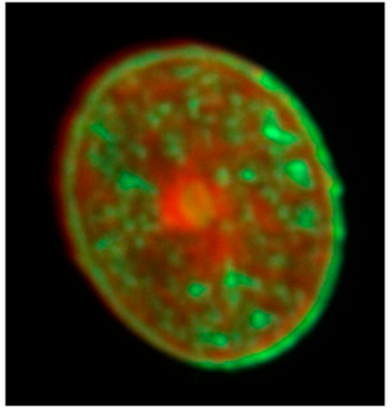

(c)

Figure 2. Fluorescently stained sections of three typical hairs having different bending modulus. (a) A section of the hair of high bending modulus (6.5 GPa); (b) a section of the hair of low bending modulus (4.7 GPa); (c) a section of the hair of which bending modulus is in the middle (5.7 GPa).

\subsection{Relationship between the Bending Modulus and the Fraction of Para-Like and Ortho-Like Cortices}

To elucidate the relationship between the bending modulus and the fraction of para-like and ortho-like cortices, it was necessary to quantify the fraction of the two cortex types. FLM was performed on the hair fibers of 27 subjects with different bending modulus (totally 69 hair fibers), and the fraction of the para-like cortex to the whole cortex $\Phi_{\mathrm{p}}$ was determined. The area of the whole cortex was determined by subtracting the cuticle and medulla areas from the whole cross sectional area. The area of para-like cortex was determined through binarization of the image. The results of $\Phi_{\mathrm{p}}$ plotted against $E$ are shown in Figure 3. There is a wide scatter in the results due to the variability of individual fibers, but they follow a significantly positive trend $\left(p=6.7 \times 10^{-4}\right.$ by Pearson's product-moment correlation test calculated with Microsoft Excel), which suggests that the more para-like cortices, the higher the bending modulus.

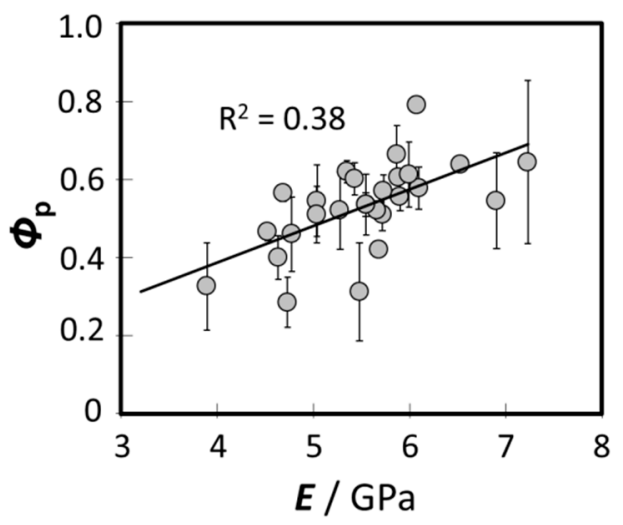

Figure 3. Fraction of the para-like cortex to the whole cortex $\left(\Phi_{\mathrm{p}}\right)$ vs. macroscopic bending modulus $E$. Circles with an error bar, mean \pm standard deviation (SD) of each subject; Solid line, result of linearization. The coefficient of determination $\mathrm{R}^{2}$ is 0.38 . 


\subsection{Modulus Inside Macrofibrils}

Figure 4a shows the result of FLM on an African-American hair with a very strong curl shape. In the figure, the arrow means the direction from the convex to the concave side of the curl. It can clearly be seen that the concave side of the hair is mostly stained by FS and the convex side mostly by SR, indicating that para-like and ortho-like cortices are distributed bilaterally in the very strong curly hair. Figure $4 \mathrm{~b}$ shows an amplitude image at a center part of the cross section of an African-American hair. Amplitude image can display the shape of the sample surface. Fine textures of macrofibrils are seen in the lower half side of the image (convex side), whereas fused macrofibrils are seen in the upper half side of it (concave side). They correspond to the patterns of ortho-like and para-like cortical cells, respectively, as was shown in TEM study by Bryson et al. [12]. Since the morphology of macrofibril was apparently different, we could understand which cortical cell is para-like or ortho-like.

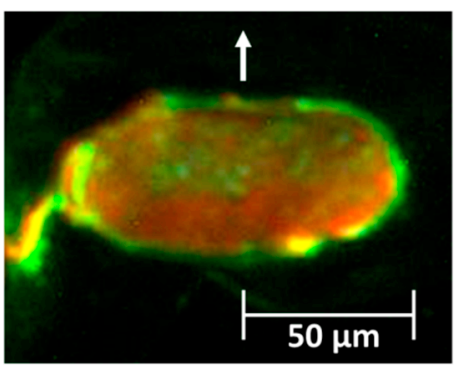

(a)

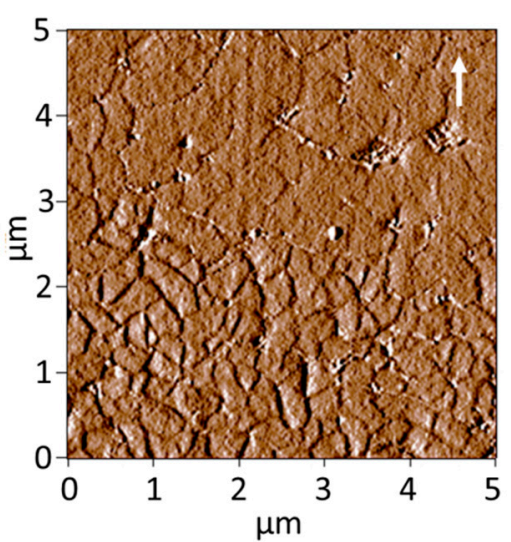

(b)

Figure 4. Observations of transverse cross sections of African-American hairs. (a) An example of a fluorescence light microscope image of a section stained with two fluorescent dyes; (b) an amplitude image $(5 \times 5 \mu \mathrm{m})$ from an atomic force microscope (AFM) at the center part of a hair. In both pictures, the arrows indicate the curvature directions from the convex to the concave side.

Figure 5a shows the histograms of elastic modulus $\left(E_{\mathrm{mf}}\right)$ inside macrofibrils determined by AFM nanoindentations for para-like and ortho-like cortical cells for the sample same as Figure $4 \mathrm{~b}$. The averaged elastic modulus was $4.0 \pm 0.6 \mathrm{GPa}$ for the para-like cortical cells and $4.0 \pm 0.5 \mathrm{GPa}$ for the ortho-like cortical cells. Figure $5 b$ is the result for a hair of Japanese female aged 57 . In the hair, the distribution of two cortical cell types was not clearly separated bilaterally, but we could distinguish them from their amplitude images. The averaged elastic modulus was $3.8 \pm 0.5 \mathrm{GPa}$ for the para-like cortical cells and $3.7 \pm 0.5 \mathrm{GPa}$ for the ortho-like cortical cells. In the two cases described above, the values for para- and ortho-like cortical cells were almost same and there was no significant difference to explain the large difference in macroscopic bending modulus. 


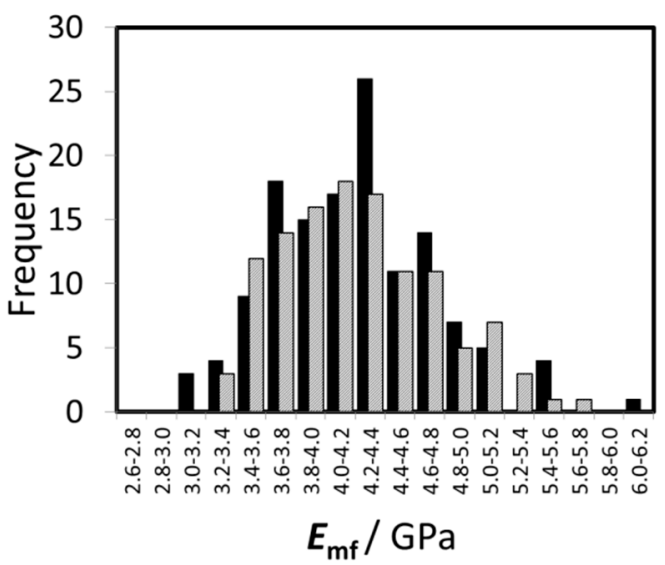

(a)

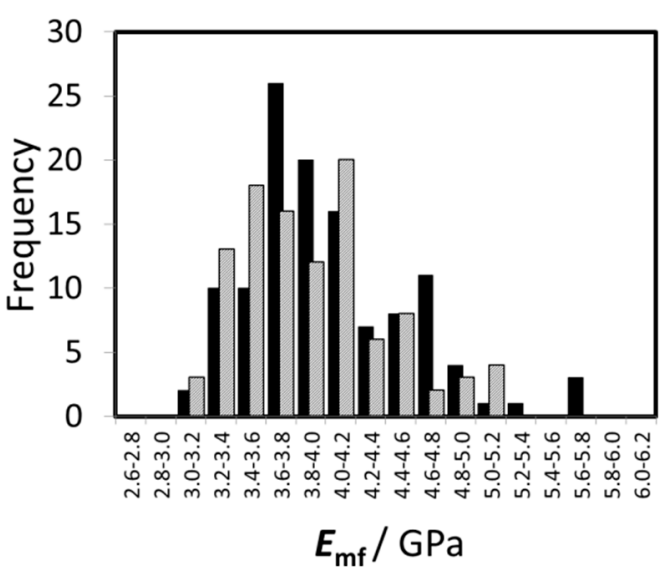

(b)

Figure 5. Histograms of elastic modulus inside macrofibrils $\left(E_{\mathrm{mf}}\right)$ evaluated by AFM nanoindentations at about $27^{\circ} \mathrm{C}$ and $65 \%$ RH. (a) an African-American hair; (b) a Japanese hair. Black bars, para-like cortical cells; shaded bars, ortho-like cortical cells. The total indentation numbers were 134 and 119 for para-like and ortho-like cortical cells in (a), respectively, and 119 and 105 for para-like and ortho-like cortical cells in (b), respectively. The averaged $E_{\mathrm{mf}}$ values of para-like and ortho-like cortical cells for the African-American hair were the same $(4.0 \mathrm{GPa})$ and those for the Japanese hair were $3.8 \mathrm{GPa}$ and 3.7 GPa, respectively.

\section{Discussion}

Macroscopic physical properties such as hair shape or stiffness should be reflected in microscopic properties such as the cells composing the hair fiber and, furthermore, those of the cell components such as proteins and their assemblies. It has previously been determined that the internal distribution of cortical cell types is attributed to the macroscopic hair shape [12] and the increase in curliness with aging relates to the increase in inhomogeneity of the cortical cell distribution [16]. In this study, we propose a relationship between macroscopic bending modulus and microscopic internal structure.

\subsection{The Effect of Cuticle}

Sogabe et al. [19] reported that the modulus of the hair cuticle was four times higher than that of the cortex and the cuticle contribution to the elasticity of a whole fiber was up to $60 \%$. However, in previous work with large numbers of hair fibers having had their cuticle scales physically removed, the bending modulus of the cuticle was found to be similar to that of the cortex [20]. Caldwell and Bryson [18] also revealed that the elastic modulus of the cuticle was similar to that of the cortex for wool fibers, using the AFM nanoindentation method. Since the cuticle component exists at the outermost region of the hair, there is no doubt that the contribution of the cuticle on bending elasticity is higher than would be expected if the whole fiber were homogeneous. Swift [21] predicted the contribution of cuticle (thickness $5 \mu \mathrm{m}$ ) to hair bending stiffness to be $30 \%-80 \%$ with a simple model. The contribution varies according to the cuticle thickness and hair major and minor diameters. In the present study, since the thickness and ratio of the cuticle for each sample was not evaluated, it is impossible to estimate the contribution of the cuticle accurately. One of the reasons why the correlation in Figure 3 is not so high could be due to the difference in the contribution of the cuticle.

\subsection{The Effect of Cortical Cell Type Distribution}

For naturally curly hair like an African-American hair, in which ortho-like and para-like cortices are clearly separated (Figure 4a), the inhomogeneous distribution would be expected to affect the bending direction and thus the hair bending modulus cannot only be expressed by the fraction of the different cortical cell types present in the hair, as indicated in Figure 3. By contrast, most Japanese hairs have less curly shapes and smaller internal inhomogeneities than African-American hairs [14]. 
As a result, with little contribution of differences in the cell distribution, the bending modulus of Japanese hair showed the simple trend that the more the para-like cortex, the higher the modulus of macroscopic properties (Figure 3).

\subsection{The Reason Why the Para-Like Cortex Is Stiffer Than the Ortho-Like Cortex}

With the force volume AFM technique on wool fiber cross sections, it was reported that the mean elastic modulus of the paracortex was 3.7 GPa and that of the orthocortex 3.3 GPa [18]. These elastic modulus values are close to the values for human hair obtained in this study. Breakspear et al. [20] examined the AFM nanoindentation study on Japanese female hairs, and reported that the elastic modulus of the cortex part varied from 3.6 GPa to $0.7 \mathrm{GPa}$ according to the RH, and was $1.9 \mathrm{GPa}$ at $27^{\circ} \mathrm{C}$ and $65.5 \% \mathrm{RH}$. The cortex type is not known but this result is smaller than the values of the present study. This difference could be due to a combination of the individual differences in hair properties being considerable and the determination of modulus is being appreciably affected by the experimental conditions such as the cantilever type used, etc. Despite such variation of elastic modulus determined by AFM nanoindentation under different conditions, it is reasonable to expect that, if the measurements are performed on the same hair cross section under the same conditions, it should be possible to directly compare the elastic modulus values from the two parts without influence from inter-fiber differences or experimental condition variations. With a similar consideration, it is also suggested that there is no significant difference between the two cortical cell types in elastic modulus inside macrofibrils composed of IFs and KAPs.

This may seem inconsistent with the result that the bending modulus significantly correlated with the fraction of para-like cortical cells to the whole cortex. Three possible reasons are proposed as to why the para-like cortex is stiffer than the ortho-like cortex. The first possibility is the difference in the volume fraction of IFs and matrix. In wool fiber, the volume fraction of matrix in the paracortical cell is more than that in the orthocortical cell $[22,23]$. As for human hair, it has been found by small angle microbeam X-ray scattering measurements that the volume fraction of the matrix existing between IF-IF is richer in the para-like than that in the ortho-like cortical cells [13,14]. KAPs, constituents of the matrix, are amorphous cysteine-rich proteins and are, therefore, supposed to contain a large number of intra- and inter-molecular cross linkages [24]. By amino acid analyses of human curly hairs, it has been found that the concave side of the curl has higher cysteine content than the convex side, meaning that cysteine is richer in para-like cortical cells [11]. Furthermore, in the case of wool, it has been shown that cysteine rich KAP1, 2, and 3 families are expressed in both cell types but the KAP4 family, ultra-high sulfur proteins, is only expressed in the paracortical cell, while high glycine tyrosine proteins are more abundant in the orthocortical cell [3]. These facts suggest that the degree of cross linkages is higher in the para-like cortical cell and this may make the para-like cortex stiffer.

The second possibility is the difference in IF arrangement in the cortical cells. In the para-like cortical cell, IFs mostly align in parallel to the fiber axis, and hence, it may be necessary to extend IF molecules that consist of $\alpha$-helices in order to extend a para-like cortical cell longitudinally. In the ortho-like cortical cell, on the other hand, the IFs align helically, therefore, longitudinal deformation can occur by rearranging the IF's tilt angle. That is, in the ortho-like cortical cell, the IF molecule itself does not have to be deformed and, instead, the deformation stress is absorbed just by the changing tilt angle. As a result, the resistance against the deformation is less and, consequently, the elastic modulus becomes smaller for the ortho-like cortices than those for the para-like ones.

The third possibility is the difference in morphology of macrofibrils. In the ortho-like cortical cell, macrofibrils are separated from one another and each is surrounded by intermacrofibrillar materials (IMM) [12]. In the para-like cortical cell, on the other hand, the macrofibrils seem to be fused and less IMM is observed. The components of IMM are thought to be residues from cytoplasm and nuclear remnants [3] (p. 88). Kitano et al. [25] showed that IMM is softer than macrofibrils in air at $25^{\circ} \mathrm{C}$ and $37 \% \mathrm{RH}$ by the force modulation method of AFM. Since IMM has no regular structure and is easily deformable, the ortho-like cortical cell has lower elastic modulus than the para-like cortical cell. 
The size of the AFM cantilever tip is larger than the diameter of IFs, while smaller than that of macrofibrils. The results by AFM nanoindentation should mainly reflect the elasticity of macrofibrils composed of IFs and KAPs and do not directly reflect that of cortical cells, the assemblies of macrofibrils. The first and second possibilities described above relate to the properties of macrofibrils and may affect the AFM nanoindentation result. However, since the differences in modulus by AFM nanoindentation were quite small between the para-like and ortho-like cortical cells, the first and second possibilities are not feasible due to the reason of the larger difference in macroscopic bending modulus. In contrast, the third possibility, which reflects the properties of the assemblies of macrofibrils, cortical cells, is the most reasonable. In order to elucidate the mechanism, it would be necessary to measure the modulus of cortical cells and so determine the contribution of IMM. However, there are no currently available methods to do this.

\subsection{The Decrease in Hair Volume with Aging and a Potential Approach to Solve It}

Hair thinning is a critical issue for aged persons. In male hair thinning, the hair miniaturization occurs at the front or the crown of the head and it is caused by the shortening of the hair growing period [26]. On the other hand, in female hair thinning, the diffuse reduction in hair density occurs over the top of the head $[27,28]$. Although most cases do not reach the diagnosis of hair thinning or hair loss, many Japanese women in over their 50s are conscious of the hair volume reduction. The main causes for this could be the decreases in hair fiber thickness [29] and in fiber numbers [28]. From the data obtained in this study, $E$ was independent of age (Figure 1 ). However, even if the value of $E$ has no correlation with the decrease in hair volume by aging, in view of the phenomenon of there being a twofold difference in bending modulus, increasing the fraction of the para-like cortical cell would be a possible approach to improving hair volume for them.

The fractions and the distribution of the para-like and ortho-like cortical cells are likely to be controlled by gene expression in hair follicles. Li et al. [30] suggested that wool fibers of the felting luster mutation found in Merino sheep consisted of only paracortical cells. The mutant wool is shiny and lacks crimp. These changes seem to be attributed to the decrease in inhomogeneous distributions of cortical cell types due to the lack of the orthocortex. As for elastic modulus of the mutant fibers, however, no data were shown. In the case of the mutant wool comprising only paracortex, extreme down regulation of three genes, encoding high glycine/tyrosine proteins that are rich in orthocortex, was found [30]. In the case of human hairs, if the difference in gene expressions in hair follicles between the hair rich in the para-like cortex and the hair rich in the ortho-like cortex is elucidated, it will be an important finding for the technology to control the hair stiffness and hair volume from the living part of the hair.

\section{Conclusions}

Hair bending modulus depends on the fractions of the constitutional cortical cell types. The hair of a higher fraction of para-like cortical cells has higher bending modulus. The contribution of the intermacrofibrillar material is plausible as a cause of the difference in the modulus of the cortical cell types. Further investigation, however, is required to completely understand the macroscopic modulus from a microscopic point of view.

Author Contributions: Conceptualzation, S.N. and T.I.; methodology, S.N.; investigation, Y.E., A.M., S.I. and T.I.; writing; T.I.; supervision, K.K.

Funding: This research received no external funding.

Acknowledgments: The authors express their gratitude to Warren G. Bryson, formerly of Canesis Network Limited, and Jolon Dyer of AgResearch Limited for useful discussions. We thank Steven Breakspear of Kao Germany $\mathrm{GmbH}$ for useful discussions and his kind editing of English for this publication.

Conflicts of Interest: The authors declare no conflict of interest. 


\section{References}

1. Parry, D.A.D.; Steinert, P.M. Intermediate filaments: Molecular architecture, assembly, dynamics and polymorphism. Q. Rev. Biophys. 1999, 32, 99-187. [CrossRef] [PubMed]

2. Robbins, C.R. Chemical and Physical Behavior of Human Hair, 5th ed.; Springer: Berlin/Heidelberg, Germany, 2012; pp. 53-63. ISBN 978-3-642-25611-0.

3. Powell, B.C.; Rogers, G.E. The role of keratin proteins and their genes in the growth, structure and properties of hair. In Formation and Structure of Human Hair; Jollès, P., Zahn, H., Höcker, H., Eds.; Birkhäuser Verlag: Basel, Switzerland, 1997; pp. 59-148. ISBN 3-7643-5119-5.

4. $\quad$ Rogers, M.A.; Langbein, L.; Praetzel-Wunder, S.; Hermelita, W.; Schweizer, J. Human Hair Keratin-Associated Proteins (KAPs). Int. Rev. Cytol. 2006, 251, 209-263. [CrossRef] [PubMed]

5. Mercer, E.H. The heterogeneity of the keratin fibers. Text. Res. J. 1953, 23, 388-397. [CrossRef]

6. Horio, M.; Kondo, T. Crimping of wool fibers. Text. Res. J. 1953, 23, 373-386. [CrossRef]

7. Rogers, G.E. Electron Microscopy of Wool. J. Ultrastruct. Res. 1959, 2, 309-330. [CrossRef]

8. Bryson, W.G.; Mastronarde, D.N.; Caldwell, J.P.; Nelson, W.G.; Woods, J.L. High voltage microscopical imaging of macrofibril ultrastructure reveals the three-dimensional spatial arrangement of intermediate filaments in Romney wool cortical cells-A causative factor in fibre curvature. In Proceedings of the 10th International Wool Textile Research Conference, Aachen, Germany, 26 November-1 December 2000.

9. Swift, J.A. Morphology and histochemistry of human hair. In Formation and Structure of Human Hair; Jollès, P., Zahn, H., Höcker, H., Eds.; Birkhäuser Verlag: Basel, Switzerland, 1997; pp. 149-175. ISBN 3-7643-5119-5.

10. Thibaut, S.; Barbarat, P.; Leroy, F.; Bernard, B.A. Human hair keratin network and curvature. Int. J. Dermatol. 2007, 46 (Suppl. 1), 7-10. [CrossRef]

11. Nagase, S.; Tsuchiya, M.; Matsui, T.; Shibuichi, S.; Tsujimura, H.; Masukawa, Y.; Satoh, N.; Itou, T.; Koike, K.; Tsujii, K. Characterization of curved hair of Japanese women with reference to internal structures and amino acid composition. J. Cosmet. Sci. 2008, 59, 317-332.

12. Bryson, W.G.; Harland, D.P.; Caldwell, J.P.; Vernon, J.A.; Walls, R.J.; Woods, J.L.; Nagase, S.; Itou, T.; Koike, K. Cortical cell types and intermediate filament arrangements correlate with fiber curvature in Japanese human hair. J. Struct. Biol. 2009, 166, 46-58. [CrossRef]

13. Kajiura, Y.; Watanabe, S.; Itou, T.; Iida, A.; Shinohara, Y.; Amemiya, Y. Structural analysis of single wool fibre by scanning microbeam SAXS. J. Appl. Cryst. 2005, 38, 420-425. [CrossRef]

14. Kajiura, Y.; Watanabe, S.; Itou, T.; Nakamura, K.; Iida, A.; Inoue, K.; Yagi, N.; Shinohara, Y.; Amemiya, Y. Structural analysis of human hair single fibres by scanning microbeam SAXS. J. Struct. Biol. 2006, 155, 438-444. [CrossRef]

15. Mamada, A.; Nakamura, K. A study of the volume and bounce decrease in hair with aging using bending elasticity measurements. J. Cosmet. Sci. 2007, 58, 485-494.

16. Nagase, S.; Kajiura, Y.; Mamada, A.; Abe, H.; Shibuichi, S.; Satoh, N.; Itou, T.; Shinohara, Y.; Amemiya, Y. Changes in structure and geometric properties of human hair by aging. J. Cosmet. Sci. 2009, 60, 637-648. [CrossRef] [PubMed]

17. ImageJ. Available online: https:/ /imagej.nih.gov/ij/ (accessed on 4 August 2006).

18. Caldwell, J.P.; Bryson, W.G. Elastic modulus mapping of the wool fibre cellular structure by atomic force microscopy. In Proceedings of the 11th International Wool Textile Research Conference, Leeds, UK, 4-9 September 2005.

19. Sogabe, A.; Yasuda, M.; Noda, A. Physical properties of human hair. 1. Evaluation of bending stress by measuring the major and the minor axis of human hair. J. Soc. Cosmet. Chem. Jpn. 2002, 36, 207-216. [CrossRef]

20. Breakspear, S.; Mamada, A.; Itou, T.; Noecker, B. Contribution of the cuticle to the stiffness of human hair: Significant or minor? IFSCC Mag. 2015, 18, 25-34.

21. Swift, J.A. Some simple theoretical considerations on the bending stiffness of human hair. Int. J. Cosmet. Sci. 1995, 17, 245-253. [CrossRef]

22. Rogers, G.E. Electron microscope studies of hair and wool. Ann. N. Y. Acad. Sci. 1959, 83, 378-399. [CrossRef] [PubMed]

23. Dobb, M.G. Electron-diffraction studies of keratin cells. J. Text. Inst. 1970, 61, 232-234. [CrossRef] 
24. Fraser, R.D.B.; MacRae, T.P.; Sparrow, L.G.; Parry, D.A.D. Disulphide bonding in $\alpha$-keratin. Int. J. Biol. Macromol. 1988, 10, 106-112. [CrossRef]

25. Kitano, H.; Yamamoto, A.; Niwa, M.; Fujinami, S.; Nakajima, K.; Nishi, T.; Naito, S. Young's modulus mapping on hair cross-section by atomic force microscopy. Compos. Interfaces 2009, 16, 1-12. [CrossRef]

26. Hamilton, J.B. Patterned loss of hair in man: Types and incidence. Ann. N. Y. Acad. Sci. 1951, 53, 708-728. [CrossRef]

27. Ludwig, E. Classification of the types of androgenetic alopecia (common baldness) occurring in the female sex. Br. J. Dermatol. 1977, 97, 247-254. [CrossRef] [PubMed]

28. Birch, M.P.; Messenger, J.F.; Messenger, A.G. Hair density, hair diameter and the prevalence of female pattern hair loss. Br. J. Dermatol. 2001, 144, 297-304. [CrossRef] [PubMed]

29. Ootsuka, H.; Nemoto, T. Study on Japanese hair. Koshokaishi 1988, 12, 192-197.

30. Li, S.W.; Ouyang, H.S.; Rogers, G.E.; Bawden, C.S. Characterization of the structural and molecular defects in fibres and follicles of the merino felting lustre mutant. Exp. Dermatol. 2008, 18, 134-142. [CrossRef] [PubMed]

(C) 2019 by the authors. Licensee MDPI, Basel, Switzerland. This article is an open access article distributed under the terms and conditions of the Creative Commons Attribution (CC BY) license (http:/ / creativecommons.org/licenses/by/4.0/). 